\title{
Outcomes of the State-of-the-art Symposium: status, challenges and future directions of offsite construction
}

\author{
Andriel FENNER ${ }^{1}$, Mohamad RAZKENARI ${ }^{1}$, Alireza SHOJAEI ${ }^{2}$, Hamed HAKIM ${ }^{1}$, Charles \\ KIBERT $^{* 3}$ \\ ${ }^{1}$ Ph.D. student, Department of Design, Construction, and Planning, University of Florida \\ ${ }^{2}$ Ph.D., Department of Design, Construction, and Planning, University of Florida \\ ${ }^{3}$ Professor, M.E. Rinker Sr. School of Construction Management, University of Florida \\ ${ }^{*}$ Corresponding author's e-mail: ckibert@ufl.edu
}

\begin{abstract}
Offsite construction is facing slow adoption despite the fact it can be a suitable solution for addressing historical problems faced by the construction industry, such as labor shortages, construction safety, time and costs overrun, and waste. The controlled factory environment creates room for innovation similar to the techniques used in the manufacturing industry. Yet, as always, the progress and adaptation of innovative ideas are challenging in the construction sector. A growing role for off-site construction requires further research and development. More collaborative efforts, industry meetings, and academic symposia are needed to bring together different disciplines and bridge the current information gap. This paper aims to present the outcomes of the "Symposium on the State-of-the-Art of Modular Construction" held in Gainesville, Florida from May $4^{\text {th }}$ to $5^{\text {th }}, 2017$ that aimed to bring together major stakeholders in modular construction. It includes an analysis of the lectures and the survey that was distributed to industry and academic experts during the symposium. Also, it investigates the state-of-the-art of modular construction and focuses on the strengths, weaknesses, opportunities, and threats that come with this building technique.
\end{abstract}

\section{KEYWORDS}

Modular construction, offsite construction, prefabrication, SWOT analysis

\section{INTRODUCTION}

The construction sector has been historically one of the most inefficient industries. In addition to the common problems of costs overruns and scheduling, projects are also generally unsustainable from the natural resources perspective and do not commonly integrate technology efficiently. The peculiarities of each project are also an obstacle to increase efficiency. Not only is the project site different for each building, but each project also has its own peculiarities related to program, design, and building products. Furthermore, the construction industry is highly influenced by external factors such as the economy, fluctuations in labor, and weather conditions. Offsite construction methods represent an immense opportunity for controlling most of these construction problems while increasing efficiency and potentially reducing costs. However, despite all its advantages, offsite construction still has had a very little market share. 
Although prefabrication has recently emerged as a Modern Method of Construction (MMC) trend, the use of prefabrication has its roots back in the process of colonization in the $16^{\text {th }}$ century, when settlers manufactured components inland and shipped them with expeditions to provide for rapid construction process of essential buildings. Offsite construction has also been largely used during and after World War II and has inspired architects in the second half of the twentieth-century (Smith \& Quale, 2017). Most of these projects failed in terms of practice and performance, leaving a negative perception in the public, housing market, and architects. Currently, the industry is trying to overcome this negative stigma by improving construction methods, materials, and even trying to develop new definitions for the industry (Goodier \& Gibb, 2007; Samuelsson Brown, Parry, \& Howlett, 2003).

In current terms, prefabrication is defined as the practice of manufacturing components of a structure in a factory and transporting the complete or semi-complete assemblies to the construction site. The scope of prefabrication ranges from the production of individual components to the complete building. Permanent Modular Construction (PMC) is a recent term used to define the process of design and prefabrication of at least 60 to 80 percent of self-standing volumetric modules offsite and under controlled factory conditions, followed by the transportation to the site for assembly (MBI, 2016). Despite modular construction being a sub-segment of the prefabrication industry, PMC benefits from the use of small prefabricated components.

While prefabricated components are extensively used in the industry, the use of volumetric modular units have not been as successful (Smith \& Quale, 2017). Currently, the modular construction industry holds around 3.5 percent of the construction market share in the US (MBI, 2016). As stated by several studies, the major drivers to use modularity are time, cost and quality. According to MBI (MBI, 2016), most modular projects can reduce 30 to 50 percent of the construction schedule. While construction costs are likely competitive to an onsite construction process, the time savings accrued from the simultaneous scheduling of offsite and onsite work enables clients to turn profits quicker and save money spent on employee displacement. Modularity also has immense potential to integrate the use of technologies and information systems, enhancing productivity and quality control by superior supply management and data integration (Kibert et al., 2016). Due to recent technology developments, modular projects have also become a potential approach for affordable, quick, and efficient construction method for reconstruction in devastated areas (Fenner, Raskenari, Hakim, \& Kibert, 2017). Furthermore, the construction process could significantly reduce the environmental impacts generated by conventional construction, especially because of the potential to minimize material waste (Kamali \& Hewage, 2017; Kamali \& Hewage, 2016). There are also opportunities to improve sustainability in all phases of the prefabricated process, but mainly during the design and manufacturing processes (Fenner \& Kibert, 2016). Lastly, modular construction has also become an alternative method for the construction to overcome the increasing problem of qualified construction labor scarcity (Kibert, Chini, \& Rumpf-Monadizadeh, 2016; Sullivan, Kibert, Fenner, \& Morque, 2017).

However, despite all the advantages and the increasing interest in offsite technologies and innovative building processes from industry advisors and experts, the use of these methods in the US still remains behind other similar economies. As part of the business development, several strategies were suggested to increase the use of modular construction in the industry. Among them, the increase in research, marketing, and professional development workshops have become a priority in the last few years for main organizations, such as the Modular Building Institute (MBI). In order to achieve higher market shares, collaborative efforts among industry and academia are still needed to bridge the current information gap. Therefore, this paper aims to present the 
outcomes of a recent symposium that aimed to bring together major stakeholders in modular construction. The results of the conference along with the survey results were synthesized and used to create a diagram of the strengths, weaknesses, opportunities, and threats (SWOT) of the modular industry.

\section{METHODS}

A Strengths, Weaknesses, Opportunities, Threats (SWOT) diagram was generated by analyzing lectures, interviews, and surveys distributed during the "Symposium on the State-ofthe-Art of Modular Construction" held in Gainesville, Florida from May $4^{\text {th }}$ to $5^{\text {th }}, 2017$. The Symposium was designed to provide a snapshot of the cutting edge of modular building design, component manufacturing, and construction techniques and to help stimulate a dialogue among the stakeholders of the modular process. The target audience for the Symposium included owners investing in modular strategies, construction management firms, architects, engineers, building component manufacturers, and subcontractors engaged in multi-trade prefabrication.

\section{RESULTS AND DISCUSSION}

The SWOT analysis is a structured planning method that identifies the internal and external factors that are favorable and unfavorable for a specific industry market. The strengths and weaknesses are internal factors defined as the characteristics of the business that give it an advantage and disadvantage over other industries, respectively. The opportunities and threats are external environmental factors not controlled by the industry that can be beneficial or could potentially damage the business, respectively. The results of a SWOT analysis is commonly used for decision-making processes and to develop an effective organizational strategy. Table 1 and Table 2 summarizes SWOT analysis from the State-of-the-art Symposium, while the following sections will further discuss each category.

\section{Strengths}

Time and costs saving are repeatedly used as the main strengths of offsite construction. The main reason lies in the fact that modules are produced in parallel with onsite operations. This reflects on a substantial reduction of the construction schedule, which is estimated to be around 30 to 50 percent depending on the project scope (2017). While construction costs are likely competitive to an onsite construction process, the time savings accrued from the simultaneous scheduling of offsite and onsite work enables clients to turn profits quicker and save money spent on employee displacement. The cost and schedule certainty is also a characteristic of modular construction. Not only are the construction schedule and costs more precisely estimated than when using conventional methods, but there is also a higher level of transparency associated with the improved supply chain management.

Offsite construction has also several benefits associated with its peculiar work environment. The most significant benefits are regarding the increased product quality and workability by protecting products and workers from adverse weather conditions. Additionally, the pre-established layout for each activity extends the benefits to other fields. At the product level, the controlled environment promotes the use of higher precision machinery. Automated and/or repeated functions reduces unnecessary moves in the factory and the number of exhaustive activities performed by workers, improving workforce productivity. There is also an improved physical ability after a workday compared to conventional construction, improving the well-being of workers and supporting family sustainability. Furthermore, workforce safety is substantially 
reduced at the factory level compared to conventional construction, especially because of the reduced volume of activities at height and open-flame jobs, better activity layout, and improved ergonomic zone. The pre-established factory layout also improves supply chain management and logistics. The organized factory layout provides an excellent opportunity for improved waste management strategies. Material waste is substantially reduced because most of the materials are bought in bulk and due to the high precision machinery. Thus, the left-over materials can also be potentially used for different modules or projects. Additionally, the lack of exposure to external weather conditions provides an excellent opportunity to recycle/reuse materials.

Modular construction popularity and demand has also increased substantially in the last years. Not only the industry has become more active and strengthened its marketing and advertisement, but the business also became more structured, meaning that the industry is working collaboratively to advance the market share. The Integrated Project Delivery (IPD) has become one of the industry's unique selling points, requiring the involvement of several AEC professionals in the project such as architects, engineers, contractors/subs, and manufactures. Projects also benefit by using IDP due to the reduced number of change orders and further design/construction problems.

\section{Weaknesses}

The issues identified in weakness section of the SWOT analysis covers those that have a negative impact on the modular construction growth. In other words, weaknesses put modular construction at a disadvantage compared to other methods available. The first issue identified was lack of innovation. This is a well-documented issue in the whole construction industry and has its root back in the risk aversiveness culture of the industry. Modular construction, in itself, is an innovative approach but its progress suffers from lack of innovation and an overall low sophistication of the modular building industry. This could be due to the fact that it is in its early stages of development and is not mature enough.

An inherent weakness of modular construction is its design limitations. Due to the production process implemented in modular construction, it poses some limitations on the designers and builders that could weaken the proliferation of the modular construction, for example, double floors and walls, lateral systems, modules cutoffs are among the design complications involved in modular construction. Also, the design should be locked as early as possible so that the modules fabrication can start. This leads to the need for earlier design decisions and a resistance to change orders. The lack of proper design, regulatory and contractual frameworks to support modular construction also weakens the adaptation of modular construction. This construction method needs new delivery and integrated practices. Underinformed management and, in general, lack of construction industry knowledge and understanding about the construction technique damage the extent that modular construction is considered an option in early project planning. These issues along with poor management and coordination in construction companies call for cultural reform. The lack of expertise and standard practices led to lower productivity in the fabrication workshops.

There is a difference between working on a conventional construction project as a subcontractor and working on a factory with repetitive modules. Understanding these issues and exploiting them could significantly improve modular construction production output. Building modules in a factory and shipping them to the job site for assembly poses inevitable restrictions on the size, weight, and extent of the modules, which essentially translate to design limitations. Shipping building modules is also a critical and tricky task that impacts the demand for modular 
construction. Module transportation, crane capacity, and the costs of cranes and module storage can cause practical and logistic issues which, in some cases, may make the whole modular project unfeasible.

Furthermore, factories for modular fabrication need high levels of capital investment which poses new challenges to the widespread use of modular construction, especially because most construction companies are small-medium size. Also, compared to conventional construction methods, modular construction requires a higher up-front cost in the project lifecycle as the construction happens early and finishes very fast, potentially causing cash flow problems for the company. Finally, the current low market share and low diversity of product types hinder the proliferation of modular construction. Strong marketing strategies and introducing new products to the market can improve this weakness.

Table 1 - Summary of major strengths and weaknesses of the modular construction industry as by 2017. Adapted from (Fenner, Zoloedova, \& Kibert, 2017)

\begin{tabular}{|c|c|}
\hline \multicolumn{2}{|c|}{ INTERNAL FACTORS } \\
\hline STRENGTHS (+) & WEAKNESSES (-) \\
\hline $\begin{array}{l}\text { - } \text { Time-saving } \\
\text { - } \text { Cost saving } \\
\text { - } \quad \text { Time and costs certainty } \\
\text { - } \text { Controlled environment production } \\
\text { - } \quad \text { Workforce productivity and wellbeing } \\
\text { - Improved quality } \\
\text { - Improved safety } \\
\text { - } \quad \text { Better logistics } \\
\text { - Improved waste management } \\
\text { - } \text { Marketing advantage } \\
\text { - Integrated project processes }\end{array}$ & $\begin{array}{l}\text { - } \text { Lack of innovation } \\
\text { - } \text { Design limitations } \\
\text { - } \text { Resistance to change orders } \\
\text { - Under informed management } \\
\text { - } \text { Lack of expertise } \\
\text { - } \text { Transportation and shipping } \\
\text { difficulties } \\
\text { - } \text { Assembly limitations } \\
\text { - Need for initial capital } \\
\text { - Low diversity of products } \\
\text { - } \text { Low market share }\end{array}$ \\
\hline
\end{tabular}

\section{Opportunities}

Modular construction goes hand in hand with many promising external opportunities. These opportunities are noted as factors that are not inherently related to modular construction within its current characteristics but have a potential to be included and improve the efficiency of the industry. An obvious example is employing innovation and advanced technologies in design, manufacturing, transportation, and assembly processes. Manufacturing industries exceedingly benefit from advanced technologies such as information systems, additive manufacturing, 3D printing, and automation. Since modular construction ties manufacturing knowledge with the production of buildings, it can potentially ease technology transfer between these two sectors. Additionally, it is expected an emergence of innovative production techniques for optimization, efficiency, quality improvement, as well as the use of the lean concepts in prefabrication as in other manufacturing industries.

Increasing attention to sustainability in the built environment represents a substantial opportunity for modular construction. The industry is intrinsically more sustainable than conventional practices, at least on the project site and module fabrication. Generally, manufactured buildings tend to have very small impacts on the site compared to conventional construction. It also turns out that implementing sustainable strategies is facilitated by the controlled production environment of factories. The intelligent and adaptable modular platform not only improves the 
level of sustainability but also improves workforce productivity and increases the efficiency of the production line. Lastly, the costs savings from using modularity could be potentially used to increase the efficiency of the project by using materials with higher performance than required by building codes. Overall, the industry can use these sustainable aspects as opportunities for advertisement and to facilitate the achievement of green building certifications.

Modular construction is an exceptional opportunity for the industry to deal with the shortage of skilled labor, which is a repeatedly reported problem in the construction industry (Sullivan et al., 2017). In fact, many construction firms are already using prefabricated modules to address labor shortage problems in particular areas. Due to these recurrent problems, the demand for modular construction is expected to grow over the long term. In theory, the reliance on modular construction is inevitable to address significant needs of housing and infrastructure. As a result, the popularity of modular construction is expected to rise among owners and builders. In this process, the market is extending to new building types and segments such as retail, hospitality, and healthcare. Beside this, modular buildings have a significant marketing potential, which can play a significant role in increasing the market demand.

Lastly, the modular technique has the flexibility of filling small, vacant, restricted, and neglected sites in urban centers. There is a growing opportunity for modular construction to deliver flexible and innovative solutions and gain this unclaimed market. Apart from this, critical economic conditions drive the building industry toward fast delivery as well as cost reduction. In other words, the fast delivery of modular projects leads to a quick return on the initial investment.

\section{Threats}

The modular construction industry faces many external threats. The conservative construction culture is the primary threat. Historically, the construction industry has always been slow in incorporating technology or innovative solutions. The main reason may be high costs associated with the process of building and the consequent reputation that a company receives for managing the project. Not to mention that, in order to try a new construction method such as modularity, the whole design and construction process must be modified, requiring a significant amount of training.

Market volatility is often said to be a twofold threat to modular construction. When the economy crashes, the modular construction industry might face higher risks associated with its unstructured market, making many small companies to file bankruptcy. On the other hand, it is often seen that is difficult for the modular construction to compete with conventional construction methods when the economy is stable and favorable because there is no need for advanced strategies for saving economic resources.

Other than the transportation and shipment regulations, there are no special building codes for modular construction. As a consequence, building inspectors often do not completely understand the modular construction process. Also, the contract management in modular construction projects is somewhat different compared to conventional buildings. The unfamiliar contractual process and the clear lack of best contractual practices on the industry may lead to deep contractual and financial issues, especially for small companies.

Lastly, logistics can be a mixed blessing for modular construction when considering the aforementioned benefits of logistics side-by-side with the complexity of external logistics. Strictly speaking, supply chain management, transportation, and shipping are also highly associated with risk and uncertainty. 
Table 2 - Summary of major opportunities and threats of the modular construction industry as by 2017. Adapted from (Fenner et al., 2017).

\begin{tabular}{|c|c|}
\hline \multicolumn{2}{|c|}{ EXTERNAL FACTORS } \\
\hline OPPORTUNITIES (+) & THREATS (-) \\
\hline 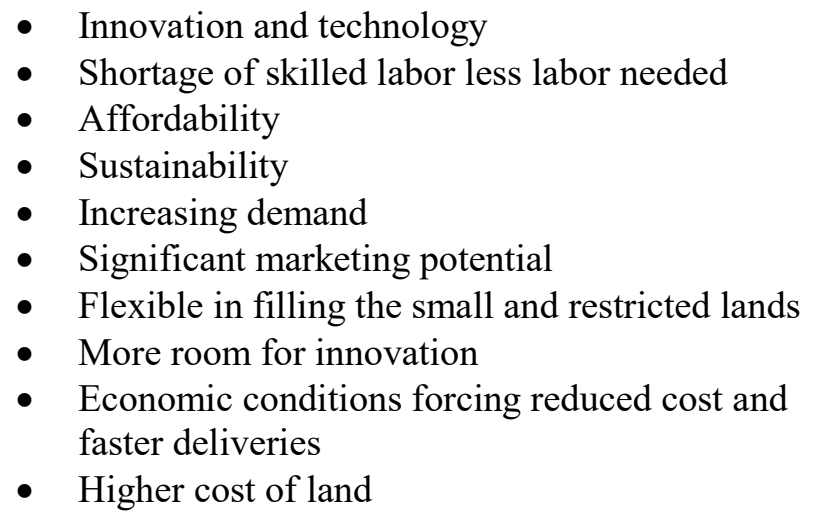 & $\begin{array}{l}\text { - Market demand changes } \\
\text { - Codes and regulations that continue } \\
\text { to restrict the use of modular } \\
\text { construction } \\
\text { - Negative perception of public and } \\
\text { industry } \\
\text { - Conservative construction culture } \\
\text { - Logistics } \\
\text { - Complex contractual process }\end{array}$ \\
\hline
\end{tabular}

\section{CONCLUSION}

Although the major drivers to use modularity are time, cost, and quality, collaborative efforts among industry and academia are still needed to bridge the current information gap. This paper has provided a SWOT analysis using the lectures, interviews, and surveys distributed during the "Symposium on the State-of-the-Art of Modular Construction" held in Gainesville, Florida from May 4th to 5th, 2017. The SWOT analysis reveals that while modular construction confronts numerous challenges to increase its role in the construction industry, the profits and gains associated with the use of modularity may outweigh the risks and deficiencies. With a closer look at the internal factors, we can see a balance in the number of strengths and weaknesses that are identified from the analysis. However, modular construction strengths might exceed its weaknesses due to the integrated benefits of the historical construction problems, including time, cost, and quality. Many of the weaknesses identified can be remedied through a better use of technologies and moving beyond the established ways of conventional construction. In other words, implementing modular construction, which is similar to the manufacturing processes in old-fashioned construction culture, is a common root for many of its weaknesses. This can be solved by transforming current culture to one closer to the manufacturing industries. Considering the external factors, the opportunities such as innovation and technology advancement are clearly superior to the identified threats, which can lead to the growth and progress of modular construction in the future. In fact, many of the identified factors can lead to both negative and positive effects, and the proper management techniques and awareness of these weaknesses and threats are necessary to keep them in the positive side and mitigate the negative impacts.

\section{ACKNOWLEDGEMENTS}

The Symposium was supported by the M.E., Sr. Rinker School of Construction Management, the College of Design, Construction, and Planning, the Engineering School of Sustainable Infrastructure and Environment, and the Powell Center for Construction and Environment at the University of Florida. We thank our colleagues who provided insight and expertise that greatly assisted the symposium and subsequent research, although they may not agree with all of the interpretations/conclusions of this paper. 


\section{References}

Fenner, A. E., \& Kibert, C. J. (2016). Sustainable manufacturing: Design and construction strategies for manufactured construction (1st Edition ed.). Gainesville: University of Florida.

Fenner, A. E., Raskenari, M. A., Hakim, H., \& Kibert, C. J. (2017). A review of prefabrication benefits for sustainable and resilient coastal areas. 6th International Network of Tropical Architecture Conference, 315-327.

Fenner, A. E., Zoloedova, V., \& Kibert, C. J. (2017). Conference report 2017: State-of-the-art of modular construction.University of Florida. 10.13140/RG.2.2.18051.60960

Goodier, C., \& Gibb, A. (2007). Future opportunities for offsite in the UK. Construction Management and Economics, 25(6), 585-595.

Kamali, M., \& Hewage, K. (2017). Development of performance criteria for sustainability evaluation of modular versus conventional construction methods. Journal of Cleaner Production, 142, 3592-3606.

Kamali, M., \& Hewage, K. (2016). Life cycle performance of modular buildings: A critical review. Renewable and Sustainable Energy Reviews, 62, 1171-1183. https://doi.org/10.1016/j.rser.2016.05.031

Kibert, C. J., Chini, A. R., \& Rumpf-Monadizadeh, S. (2016). Introduction to manufactured construction (Third Edition ed.). Gainesville: University of Florida.

Kibert, C. J., Chini, A. R., Rumpf-Monadizadeh, S., Raskenari, M. A., Fenner, A. E., Hakim, H., Garg, Y. (2016). Advanced topics in manufactured construction. Gainesville: University of Florida.

MBI. (2016). Modular advantage for the commercial modular construction industry: $P M C \& R B$ annual statistical data. ( No. 3Q).Modular Building Institute.

MBI. (2017). Why modular? Retrieved from http://www.modular.org/HtmlPage.aspx?name=why modular

Samuelsson Brown, G., Parry, T., \& Howlett, C. (2003). Offsite fabrication: UK attitudes and potential. BSRIA, 130pp,

Smith, R. E., \& Quale, J. D. (2017). Offsite architecture: Constructing the future Taylor \& Francis.

Sullivan, J., Kibert, C. J., Fenner, A. E., \& Morque, S. (2017). Florida construction workforce taskforce report. (Tech. Report). University of Florida: 10.13140/RG.2.2.13673.90728 